Nonl. Analysis and Differential Equations, Vol. 2, 2014, no. 2, 69 - 81 HIKARI Ltd, www.m-hikari.com http://dx.doi.org/10.12988/nade.2014.31225

\title{
Harnack Inequality and Continuity of Solutions for Quasilinear Elliptic Equations in Sobolev Spaces with Variable Exponent
}

\author{
Azeddine Baalal and Abdelbaset Qabil \\ Department of Mathematics - Laboratory MACS \\ Faculty of Sciences Ä̈n Chock, University of HASSAN II \\ B.P. 5366, Casablanca - Morocco
}

Copyright (C) 2014 Azeddine Baalal and Abdelbaset Qabil. This is an open access article distributed under the Creative Commons Attribution License, which permits unrestricted use, distribution, and reproduction in any medium, provided the original work is properly cited.

\begin{abstract}
We investigate regularity properties of solutions to the quasilinear elliptic equations in Sobolev spaces with variable exponent, we prove the Harnack's inequality and continuity of solutions.
\end{abstract}

Mathematics Subject Classification: 35J62, 49N60, 35J60, 35J25.

Keywords: Quasilinear elliptic equation, variable exponent, Caccioppoli estimate, Moser's iteration method, Harnack inequality

\section{Introduction}

In the present paper we study the regularity of boundary points for solutions to the quasilinear elliptic equations:

$$
-\operatorname{div} \mathcal{A}(x, \nabla u)+\mathcal{B}(x, u)=0,
$$

Our purpose is to establish the Harnack's inequality

$$
\text { ess } \sup _{B(x, R)} u \leq C\left(\text { ess } \inf _{B(x, R)} u+R\right)
$$


where $C$ is independent of $u$ and the ball $B=B\left(x_{0}, R\right)$ for $x_{0} \in \Omega, R>0$ and we prove the continuity of solutions for (1).

Harnack's inequality and other regularity results for (1) require additional assumptions on the function $\mathrm{p}($.$) ; see the counterexamples in [6]. The so$ called logarithmic Hölder continuity condition seems to be the right one for our purposes. This condition was originally introduced by Zhikov [15] in the context of the Lavrentiev phenomenon for solutions of (1), and it has turned out to be a useful tool in regularity and other applications, see, e.g., [1, 2].

For the existence and uniqueness of solutions $u \in \mathcal{W}^{1, p(x)}(\Omega)$ where $1<p(x)<$ $d$ for all $x \in \Omega$, of the variational Dirichlet problem associated with the quasilinear elliptic equation (1) see [4], these solutions are obtained by the $\mathrm{p}($.$) -$ obstacle problem.

A typical example for the operator $\mathcal{A}$ and $\mathcal{B}$ are $\mathcal{A}(x, \nabla u)=|\nabla u|^{p(x)-2} \nabla u$ and $\mathcal{B}(x, u)=|u|^{p(x)-2} u$ respectively, for all $x \in \mathbb{R}^{d}$ thus $\Delta_{p(x)} u=\operatorname{div}\left(|\nabla u|^{p(x)-2} \nabla u\right)$.

Our problem has been studied in many paper see e.g. [11, 12]. Olli Toivanen [11] proved that this problem has a solution when the operator $\mathcal{B}$ depends on $x, u, \nabla u$ where $\delta(x)=p(x)-1$. The main aim of this section is to generalize the condition on $\delta(x)$, We are interested in the case that the operator $\mathcal{B}$ depends only on $x$ and $u$, satisfying the previous hypothesis (H3), where $p(x)-1 \leq \delta(x)<p^{*}(x)$, knowing that the study of the case where $\delta$ satisfies the condition $0 \leq \delta(x)<p(x)-1$ is already investigated in several articles. The contribution of this paper is to verify the Harnack principale for a weak solutions of quasilinear elliptic equations (1) by using the trick of modified test functions under our assumptions (H3) below.

In the first section, we introduce some generalization and position of the problem. In second section we give some basic facts about variable exponent spaces and a rough overview of properties of solutions of the prototype equality. In section 3, we generalize, with detailed proofs, Harnack's inequality (2) to all quasilinear elliptic equations (1) with growth conditions of a non-standard form. In last section, we present the concluding remarks.

\section{Some preliminaries}

We start this section with some definitions and main results of Lebesgue spaces with variable exponent, and Sobolev spaces modeled upon them. For each open bounded subset $\Omega$ of $\mathbb{R}^{d}(d \geq 2)$, we define the Lebesgue space with variable exponent $L^{p(.)}(\Omega)$ as the set of all measurable functions $p: \Omega \rightarrow$ 
] $1,+\infty\left[\right.$ called a variable exponent and we denote $p^{-}:=\operatorname{ess~inf}_{x \in \bar{\Omega}} p(x)$ and $p^{+}:=\operatorname{ess}_{\sup } \in \bar{\Omega} p(x)$.

We introduce also the convex modular

$$
\varrho_{p(x)}(u)=\int_{\Omega}|u|^{p(x)} d x .
$$

If the exponent is bounded, i.e., if $p^{+}<\infty$, then the expression

$$
\|u\|_{p(.)}=\inf \left\{\lambda>0: \varrho_{p(.)}\left(\frac{u}{\lambda}\right) \leq 1\right\}
$$

defines a norm in $L^{p(.)}(\Omega)$, called the Luxemburg norm.

One central property of $L^{p(.)}(\Omega)$ is that the norm and the modular topologies coincide,i.e., $\quad \varrho_{p(.)}\left(u_{n}\right) \rightarrow 0$ if and only if $\left\|u_{n}\right\|_{p(.)} \rightarrow 0$.

We denote by $L^{p^{\prime}(.)}(\Omega)$ the conjugate space of $L^{p(.)}(\Omega)$ where $\frac{1}{p(x)}+\frac{1}{p^{\prime}(x)}=1$.

Proposition 1 (Generalized Hölder inequality [14]) For any $u \in L^{p(.)}(\Omega)$ and $v \in L^{p^{\prime}(.)}(\Omega)$, we have

$$
\left|\int_{\Omega} u v d x\right| \leq\left(\frac{1}{p^{-}}+\frac{1}{{p^{\prime}}^{-}}\right)\|u\|_{p(.)}\|v\|_{p^{\prime}(.)} .
$$

We define the variable exponent Sobolev space (see [9], [5],[8], [14]) by

$$
\mathcal{W}^{1, p(.)}(\Omega)=\left\{u \in L^{p(.)}(\Omega):\|\nabla u\| \in L^{p(.)}(\Omega)\right\} .
$$

with the norm

$$
\|u\|_{1, p(.)}=\|u\|_{p(.)}+\|\nabla u\|_{p(.)} \quad \forall u \in \mathcal{W}^{1, p(.)}(\Omega) .
$$

The local Sobolev space $\mathcal{W}_{\text {loc }}^{1, p(.)}(\Omega)$ consists of functions $u$ that belong to $\mathcal{W}_{\text {loc }}^{1, p(.)}(U)$ for all open sets $U$ compactly contained in $\Omega$. The Sobolev space with zero boundary values, $\mathcal{W}_{0}^{1, p(.)}(\Omega)$, is defined as the completion of $C_{0}^{\infty}(\Omega)$ in the norm of $\mathcal{W}^{1, p(.)}(\Omega)$.

Let $p^{*}(x)$ be the Sobolev conjugate exponent of $p(x)$ defined by

$$
p^{*}(x)= \begin{cases}\frac{d p(x)}{d-p(x)} & \text { for } p(x)<d \\ +\infty & \text { for } p(x) \geq d\end{cases}
$$

We assume further on that, there exist positive constant $C$ such that the function $p$ satisfies logarithmic Hölder continuity condition if :

$$
(\star)\left\{\begin{array}{l}
\exists C>0:|p(x)-p(y)| \leq \frac{C}{-\log |x-y|} \text { for }|x-y|<\frac{1}{2}, \\
1<p^{-} \leq p^{+}<d
\end{array}\right.
$$


Proposition 2 (The $\mathrm{p}($.$) -Poincar?e inequality)$

Let $\Omega$ be a bounded open set and let $p: \Omega \rightarrow[1, \infty[$ satisfy $(\star)$ There exists a constant $C$, depending only on $p($.$) and \Omega$, such that the inequality

$$
\|u\|_{p(.)} \leq C\|\nabla u\|_{p(.)} \quad \forall u \in \mathcal{W}_{0}^{1, p(.)}(\Omega)
$$

Lemma 2.1 (Sobolev inequality [7]) Let $\Omega$ be a bounded open set and $u$ in

$\mathcal{W}_{0}^{1, p(.)}(\Omega)$. There exists a constant $C$ such that

$$
\left(\int_{\Omega}|u|^{\frac{d p(x)}{d-1}} d x\right)^{\frac{d-1}{d p(x)}} \leq C\left(\int_{\Omega}|\nabla u|^{p(x)} d x\right)^{\frac{1}{p(x)}}
$$

\section{Proposition 3}

Assuming $p^{-}>1$, the spaces $\mathcal{W}^{1, p(.)}(\Omega)$ and $\mathcal{W}_{0}^{1, p(.)}(\Omega)$ are separable and reflexive Banach spaces.

Throughout the paper we suppose that the functions $\mathcal{A}: \mathbb{R}^{d} \times \mathbb{R}^{d} \rightarrow \mathbb{R}^{d}$ is a Carathéodory function satisfying the following assumptions:

(H1) $|\mathcal{A}(x, \xi)| \leq \beta\left[k(x)+|\xi|^{p(x)-1}\right] ;$

(H2) $\mathcal{A}(x, \xi) \xi \geq \nu|\xi|^{p(x)}$;

for a.e. $x \in \Omega$, all $\xi \in \mathbb{R}^{d}$, where $k(x)$ is a positive bounded function lying in $L^{p^{\prime}(x)}(\Omega)$ and $\beta, \nu>0$.

In this paper we suppose that the function $\mathcal{B}: \mathbb{R}^{d} \times \mathbb{R} \rightarrow \mathbb{R}$ is given Carathéodory function and the following condition is satisfied:

(H3) $|\mathcal{B}(\mathbf{x}, \zeta)| \leq \mathbf{g}(\mathbf{x})+|\zeta|^{\delta(\mathbf{x})}$

for a.e. $x \in \Omega$, all $\zeta \in \mathbb{R}^{d}$, where $g$ is a positive bounded function lying in $L^{p^{\prime}(x)}(\Omega)$ and $p(x)-1 \leq \delta(x)<p^{*}(x)$.

Remark 2.1 Under the assumption ( $\star$ ) Harnack's inequality and local Hölder continuity follow from Moser or De Giorgi-type procedure; see [10, 2, 3]. An interesting feature of this theory is that estimates are intrinsic in the sense that they depend on the solution itself. For example, supersolutions are assumed to be locally bounded and Harnack-type estimates in [2] depend on this bound.

Definition 2.1 We say that a $u \in \mathcal{W}_{\text {loc }}^{1, p(.)}(\Omega)$ is a $p($.$) -solution of (1)$ in $\Omega$ provided that for all $\varphi \in \mathcal{W}_{0}^{1, p(.)}(\Omega)$ if,

$$
\int_{\Omega} \mathcal{A}(x, \nabla u) \cdot \nabla \varphi d x+\int_{\Omega} \mathcal{B}(x, u) \varphi d x=0
$$


Definition 2.2 A function $u \in \mathcal{W}_{\text {loc }}^{1, p(.)}(\Omega)$ is termed $p($.$) -supersolutions of$ (1), if and only if, for all non-negative functions $\varphi \in \mathcal{W}_{0}^{1, p(.)}(\Omega)$ we have,

$$
\int_{\Omega} \mathcal{A}(x, \nabla u) \cdot \nabla \varphi d x+\int_{\Omega} \mathcal{B}(x, u) \varphi d x \geq 0
$$

A function $u$ is a $\mathbf{p}($.$) -subsolution in \Omega$ if $-u$ is a $\mathrm{p}($.$) -supersolution in \Omega$, and a solution in $\Omega$ if it is both a super- and a $\mathrm{p}($.$) -subsolution in \Omega$.

\section{Harnack inequality and continuity of solu- tions to quasilinear elliptic equations}

The Harnack inequality is a very important estimate in the study of $\mathrm{p}($.$) -$ solutions of quasilinear elliptic equations.

\subsection{Main result}

We start by adapting a standard Caccioppoli type estimate for $\mathrm{p}($.$) -supersolution$ of (1). Then we use the Caccioppoli estimate to show that for a fixed, nonnegative $\mathrm{p}($.$) -supersolution u$, the inequality (2).

The Harnack inequality is indispensable as a tool in the qualitative theory of second-order elliptic equations. In particular, it implies continuity of weak solutions see $[11,13]$.

By non-linearity we mean that if $p \neq 2$ then the weak solutions do not form a linear space. However the set of weak solutions is closed under constant multiplication. By celebrated De Giorgi's method and Moser's iteration the weak solutions are locally Hölder continuous and satisfy Harnack's inequality.

Remark 3.1 Our notation is rather standard. Various constants are denoted by $C$ and the value of the constant may differ even on the same line. The quantities on which the constants depend are given in the statements of the theorems and lemmas.

Lemma 3.1 Let $E$ be a measurable subset of $\mathbb{R}^{d}$. For all nonnegative measurable functions $\psi$ and $\varphi$ defined on $E$,

$$
\int_{E} \psi \varphi^{p_{E}^{-}} d x \leq \int_{E} \psi d x+\int_{E} \psi \varphi^{p(x)} d x
$$


The following Caccioppoli estimate is the key result of this paper; and it is a modification of [[7], Lemma 3.2]. The new feature in the estimate is the choice of a test function which includes the variable exponent.

Lemma 3.2 (Caccioppoli estimate)

Suppose that $u$ is a nonnegative $p($.$) -supersolution in B_{4 R}$. Let $E$ be a measurable subset of $B_{4 R}$ and $\eta \in \mathcal{C}_{0}^{\infty}\left(B_{4 R}\right)$ such that $0 \leq \eta \leq 1$. Then for every $\gamma_{0}<0$ there is a constant $C$ depending on $p$ and $\gamma_{0}$ such that the inequality:

$$
\begin{aligned}
& \int_{E} \eta^{p_{B_{4}}^{+}} u^{\gamma-1}|\nabla u|^{p^{-}} d x \leq C \int_{B_{4 R}}\left(u^{\gamma+p(x)-1}|\nabla \eta|^{p(x)}+\eta^{p_{B_{4}}^{+}} u^{\gamma-1}\right) \\
& +\left(u^{\gamma+p(x)-1} d x+\eta^{p_{B_{4 R}}^{+}-1} u^{\gamma+\delta(x)}\right) d x
\end{aligned}
$$

holds for every $\gamma<\gamma_{0}<0$ and $p(x)-1 \leq \delta(x)<p^{*}(x)$.

Proof 1 Let $\theta=p_{B_{4 R}}^{+}$. We want to test with the function $\varphi=\eta^{\theta} u^{\gamma}$.

To this end we show that $\varphi \in \mathcal{W}_{0}^{1, p(.)}\left(B_{4 R}\right)$. Since $\eta$ has a compact support in $B_{4 R}$, it is enough to show that $\varphi \in \mathcal{W}^{1, p(.)}(\Omega)$. We observe that $\varphi \in L^{p(x)}(\Omega)$ since $\left|u^{\gamma}\right| \eta^{\theta} \leq R^{\gamma}$. Furthermore, we have

$$
\nabla \varphi=\gamma \eta^{\theta} u^{\gamma-1} \nabla u+\theta \eta^{\theta-1} u^{\gamma} \nabla \eta
$$

Using the fact that $u$ is a $\mathrm{p}($.$) -supersolution and \varphi$ is a nonnegative test function we find that

$$
\begin{aligned}
0 \leq & \int_{B_{4 R}} \mathcal{A}(x, \nabla u) \cdot \nabla \varphi d x+\int_{B_{4 R}} \mathcal{B}(x, u) \varphi d x \\
& =\int_{B_{4 R}} \gamma \eta^{\theta} u^{\gamma-1} \mathcal{A}(x, \nabla u) \cdot \nabla u d x+\int_{B_{4 R}} \theta \eta^{\theta-1} u^{\gamma} \mathcal{A}(x, \nabla u) \cdot \nabla \eta d x+\int_{B_{4 R}} \mathcal{B}(x, u) \eta^{\theta} u^{\gamma} d x
\end{aligned}
$$

We denote the left-hand side of the next inequality by $I$. Since $\gamma$ is a negative number this implies by the structural conditions (H1), (H2) and (H3) that

$$
\begin{aligned}
I= & \left|\gamma_{0}\right| \nu \int_{B_{4 R}} \eta^{\theta} u^{\gamma-1}|\nabla u|^{p(x)} d x \\
& \leq \beta \int_{B_{4 R}} \theta \eta^{\theta-1} u^{\gamma}\left(k(x)+|\nabla u|^{p(x)-1}\right)|\nabla \eta| d x+\int_{B_{4 R}}\left(g(x)+u^{\delta(x)}\right) \eta^{\theta} u^{\gamma} d x \\
& \leq \beta \theta \overbrace{\int_{B_{4 R}} \eta^{\theta-1} u^{\gamma}|\nabla u|^{p(x)-1}|\nabla \eta| d x}^{=I_{1}}+\beta \theta \overbrace{\int_{B_{4 R}} \eta^{\theta-1} u^{\gamma} k(x)|\nabla \eta| d x}^{=I_{2}}+\overbrace{\int_{B_{4 R}}\left(g(x)+u^{\delta(x)}\right) \eta^{\theta} u^{\gamma} d x}^{=I_{3}}
\end{aligned}
$$

Using Young's inequality, $0<\varepsilon \leq 1$, we obtain the first estimate

$$
\begin{aligned}
I_{1} \leq \int_{B_{4 R}}( & \left.\frac{1}{\varepsilon}\right)^{p(x)-1}\left(\eta^{\theta-\frac{\theta}{p^{\prime}(x)}-1} u^{\frac{\gamma+p(x)-1}{p(x)}}|\nabla \eta|\right)^{p(x)}+\varepsilon\left(\eta^{\frac{\theta}{p^{\prime}(x)}} u^{\gamma-\frac{\gamma+p(x)-1}{p(x)}}|\nabla u|^{p(x)-1}\right)^{p^{\prime}(x)} d x \\
& \leq\left(\frac{1}{\varepsilon}\right)^{\theta-1} \int_{B_{4 R}} \eta^{\theta-p(x)} u^{\gamma+p(x)-1}|\nabla \eta|^{p(x)} d x+\varepsilon \int_{B_{4 R}} \eta^{\theta} u^{\gamma-1}|\nabla u|^{p(x)} d x \\
& \leq\left(\frac{1}{\varepsilon}\right)^{\theta-1} \int_{B_{4 R}} u^{\gamma+p(x)-1}|\nabla \eta|^{p(x)} d x+\varepsilon \int_{B_{4 R}} \eta^{\theta} u^{\gamma-1}|\nabla u|^{p(x)} d x
\end{aligned}
$$


Next we estimate the last tow integrals $I_{2}$ and $I_{3}$.

To estimate the integral $I_{2}$, we denote $0 \leq v=\eta+|\nabla \eta|$ and $k$ is a positive bounded function there exist a constant $M>0$, and by Young's inequality we have

$$
\begin{aligned}
I_{2}= & \int_{B_{4 R}} \eta^{\theta-1} u^{\gamma} k(x)|\nabla \eta| d x \leq M \int_{B_{4 R}} v \eta^{\theta-1} u^{\gamma} d x \\
& \leq M\left(\left(\frac{1}{\varepsilon}\right)^{\theta-1} \int_{B_{4 R}} \eta^{\theta-p(x)} u^{\gamma+p(x)-1} v^{p(x)} d x+\varepsilon \int_{B_{4 R}} \eta^{\theta} u^{\gamma-1} d x\right) \\
& \leq M\left(\left(\frac{1}{\varepsilon}\right)^{\theta-1} \int_{B_{4 R}} u^{\gamma+p(x)-1} v^{p(x)} d x+\varepsilon \int_{B_{4 R}} \eta^{\theta} u^{\gamma-1} d x\right) \\
& \leq M\left(\left(\frac{1}{\varepsilon}\right)^{\theta-1} \int_{B_{4 R}} u^{\gamma+p(x)-1}(1+|\nabla \eta|)^{p(x)} d x+\varepsilon \int_{B_{4 R}} \eta^{\theta} u^{\gamma-1} d x\right)
\end{aligned}
$$

Using the inequality $(\varphi+\psi)^{p(.)} \leq 2^{p(.)-1}\left(|\varphi|^{p(.)}+|\psi|^{p(.)}\right), \quad$ for $\quad p() \geq$.1 ,

$$
\begin{aligned}
I_{2} \leq & \left(\frac{1}{\varepsilon}\right)^{\theta-1} \int_{B_{4 R}} u^{\gamma+p(x)-1} 2^{p(x)-1}\left(1+|\nabla \eta|^{p(x)}\right) d x+\varepsilon \int_{B_{4 R}} \eta^{\theta} u^{\gamma-1} d x \\
& \leq \frac{2^{\theta-1}}{\varepsilon^{\theta-1}} \int_{B_{4 R}} u^{\gamma+p(x)-1}\left(1+|\nabla \eta|^{p(x)}\right) d x+\varepsilon \int_{B_{4 R}} \eta^{\theta} u^{\gamma-1} d x \\
& \leq\left(\frac{2}{\varepsilon}\right)^{\theta-1} \int_{B_{4 R}} u^{\gamma+p(x)-1}|\nabla \eta|^{p(x)} d x+\left(\frac{2}{\varepsilon}\right)^{\theta-1} \int_{B_{4 R}} u^{\gamma+p(x)-1}+\varepsilon \int_{B_{4 R}} \eta^{\theta} u^{\gamma-1} d x
\end{aligned}
$$

To estimate the integral $I_{3}$, and by the assumption $g$ is a positive bounded function there exist a $N>0$ such that

$$
\begin{aligned}
I_{3}= & \int_{B_{4 R}}\left(g(x)+u^{\delta(x)}\right) \eta^{\theta} u^{\gamma} d x \leq \int_{B_{4 R}}\left(N+u^{\delta(x)}\right) \eta^{\theta} u^{\gamma} d x \\
& \leq \int_{B_{4 R}} \eta^{\theta-1} u^{\gamma+\delta(x)} d x+N \overbrace{\int_{B_{4 R}} \eta^{\theta-1} u^{\gamma} d x}^{=I_{3}^{\prime}}
\end{aligned}
$$

By Young's inequality we have

$$
I_{3}^{\prime} \leq\left(\frac{1}{\varepsilon}\right)^{\theta-1} \int_{B_{4 R}} u^{\gamma+p(x)-1} d x+\varepsilon \int_{B_{4 R}} \eta^{\theta} u^{\gamma-1} d x
$$

Thus

$$
I_{3} \leq \int_{B_{4 R}} \eta^{\theta-1} u^{\gamma+\delta(x)} d x+\left(\frac{1}{\varepsilon}\right)^{\theta-1} \int_{B_{4 R}} u^{\gamma+p(x)-1} d x+\varepsilon \int_{B_{4 R}} \eta^{\theta} u^{\gamma-1} d x
$$


Therefore

$$
\begin{aligned}
& \left(\left|\gamma_{0}\right| \nu-\theta \beta \varepsilon\right) \int_{B_{4 R}} \eta^{\theta} u^{\gamma-1}|\nabla u|^{p(x)} d x \leq \theta \beta \frac{\left(2^{\theta-1}+1\right)}{\varepsilon^{\theta-1}} \int_{B_{4 R}} u^{\gamma+p(x)-1}|\nabla \eta|^{p(x)} d x \\
+ & \varepsilon(\theta \beta+1) \int_{B_{4 R}} \eta^{\theta} u^{\gamma-1} d x+\frac{2^{\theta-1} \theta \beta+1}{\varepsilon^{\theta-1}} \int_{B_{4 R}} u^{\gamma+p(x)-1} d x+\int_{B_{4 R}} \eta^{\theta-1} u^{\gamma+\delta(x)} d x
\end{aligned}
$$

By choosing $\varepsilon=\min \left\{1, \frac{\left|\gamma_{0}\right| \nu}{2 \theta \beta}\right\}$

$$
\begin{aligned}
\int_{B_{4 R}} & \eta^{\theta} u^{\gamma-1}|\nabla u|^{p(x)} d x \leq \\
\quad \leq & C_{1} \int_{B_{4 R}} u^{\gamma+p(x)-1}|\nabla \eta|^{p(x)} d x+C_{2} \int_{B_{4 R}} \eta^{\theta} u^{\gamma-1} d x+C_{3} \int_{B_{4 R}} u^{\gamma+p(x)-1} d x+ \\
& +C_{4} \int_{B_{4 R}} \eta^{\theta-1} u^{\gamma+\delta(x)} d x
\end{aligned}
$$

Where $C_{1}=\left(2^{\theta-1}+1\right)\left(\frac{2 \theta \beta}{\left|\gamma_{0}\right| \nu}\right), C_{2}=1+\frac{1}{\theta \beta}, C_{3}=\left(2^{\theta-1}+\frac{1}{\theta \beta}\right)\left(\frac{2 \theta \beta}{\left|\gamma_{0}\right| \nu}\right)^{\theta}$, and $C_{4}=\frac{2}{\left|\gamma_{0}\right| \nu}$.

We take $C=C_{i}$ for $i=1,2,3,4$. and we have

$$
\begin{aligned}
\int_{B_{4 R}} \eta^{p_{B_{4 R}}^{+}} u^{\gamma-1}|\nabla u|^{p(x)} d x \leq C & \int_{B_{4 R}}\left(u^{\gamma+p(x)-1}|\nabla \eta|^{p(x)}+\eta^{p_{B_{4 R}}^{+}} u^{\gamma-1}\right) \\
& +\left(u^{\gamma+p(x)-1} d x+\eta^{p_{B_{4}}^{+}-1} u^{\gamma+\delta(x)}\right) d x
\end{aligned}
$$

By the lemma 3.1 we obtain

$$
\int_{B_{4 R}} \eta^{\theta} u^{\gamma-1}|\nabla u|^{p^{-}} d x \leq \int_{B_{4 R}} \eta^{\theta} u^{\gamma-1} d x+\int_{B_{4 R}} \eta^{\theta} u^{\gamma-1}|\nabla u|^{p(x)} d x
$$

and using the previous inequality we obtain the claim.

$$
\begin{aligned}
& \int_{E} \eta^{p_{B_{4 R}}^{+}} u^{\gamma-1}|\nabla u|^{p^{-}} d x \leq C \int_{B_{4 R}}\left(u^{\gamma+p(x)-1}|\nabla \eta|^{p(x)}+\eta^{p_{B_{4 R}}^{+}} u^{\gamma-1}\right) \\
& +\left(u^{\gamma+p(x)-1} d x+\eta^{p_{B_{4 R}}^{+}-1} u^{\gamma+\delta(x)}\right) d x
\end{aligned}
$$

So the proof of lemma is achieved.

\subsection{Weak Harnack Inequality}

In this section we prove a weak Harnack inequality for $\mathrm{p}($.$) -supersolutions to$ (1). 
Throughout this subsection we write $v=u+R$ where $u$ is a nonnegative $\mathrm{p}($.)-supersolution and $0 \leq R \leq 1$.

We start by the following technical lemma that is need later. These results are mainly from [7]

Lemma 3.3 If the exponent $p($.$) is log-Hölder continuous, then R^{-p(x)} \leq$ $C R^{-p_{E}^{-}}$where $x \in E \subset B_{R}$ and $R>0$.

Lemma 3.4 Let $f$ be a positive measurable function and assume that the exponent $p($.$) is log-Hölder continuous. Then$

$$
\int_{B_{r}} f^{p_{B_{4 R}}^{+}-p_{B_{4 R}}^{-}} d x \leq C\|f\|_{L^{s}\left(B_{r}\right)}^{p_{B_{4 R}}^{+}-p_{B_{4 R}}^{-}}
$$

for any $s>p_{B_{4 R}}^{+}-p_{B_{4 R}}^{-}$

Now we have everything ready for the iteration. We write

$$
\Phi\left(f, q, B_{r}\right)=\left(\int_{B_{r}} f^{q} d x\right)^{\frac{1}{q}}
$$

for a nonnegative measurable function $f$.

The point is that the Moser iteration technique used in [7] remains valid under our consideration.

Lemma 3.5 Let $u$ is a nonnegative $p($.$) -supersolution of (1) in B_{4 R}$ and let $R \leq \rho<r \leq 3 R$. Then the inequality

$$
\Phi\left(v, q \tau, B_{r}\right) \leq C^{\frac{1}{|\tau|}}(1+|\tau|)^{\frac{p_{B_{4 R}}^{+}}{|\tau|}}\left(\frac{r}{r-\rho}\right)^{\frac{p_{B_{4 R}}^{+}}{|\tau|}} \Phi\left(v, \tau \frac{d}{d-1}, B_{\rho}\right)
$$

holds for every $\tau<0$ and $1<q<\frac{d}{d-1}$. The constant $C$ depends on $d, p$, and the $L^{q^{\prime} s}\left(B_{4 R}\right)$-norm of $u$ with $s>p_{B_{4 R}^{+}}^{+}-p_{B_{4 R}}^{-}$and all structure constants and functions of (H1),(H2) and (H3) hypothesis .

Proof 2 Let $\theta^{+}=p_{B_{4 R}}^{+}$and $\theta^{-}=p_{B_{4 R}}^{-}$, we take $\gamma=\tau-\theta^{-}+1$. In (4) of the lemma 3.2 we have

$$
\begin{gathered}
\int_{B_{4 R}} \eta^{\theta^{+}} v^{\tau-\theta^{-}}|\nabla u|^{\theta^{-}} d x \\
\leq C \int_{B_{4 R}} \overbrace{\left(\eta^{\theta^{+}} u^{\tau-\theta^{-}}+u^{\tau-\theta^{-}+p(x)}|\nabla \eta|^{p(x)}\right)}^{=I_{1}}+\overbrace{\left(u^{\tau-\theta^{-}+p(x)}+\eta^{\theta^{+}-1} u^{\tau-\theta^{-}+1+\delta(x)}\right)}^{=I_{2}} d x
\end{gathered}
$$

Now we take the test function $\eta \in \mathcal{C}_{0}^{\infty}\left(B_{r}\right)$ with $0 \leq \eta \leq 1, \eta=1$ in $B_{\rho}$, and

$$
|\nabla \eta| \leq \frac{C r}{R(r-\rho)}
$$


Next we went to estimate the integral $I_{2}$ by the integral

$$
\left(\int_{B_{r}} v^{q \tau} d x\right)^{\frac{1}{q}}
$$

Using lemma 3.3,3.4, the first integral $I_{1}$ is estimated by (7) see [7].

Finally, for the second integral $I_{2}$ we have by Hölder's inequality

$$
\begin{aligned}
\int_{B_{r}} & u^{\tau-\theta^{-}+p(x)} d x \leq \int_{B_{r}} v^{\tau-\theta^{-}+p(x)} d x \\
& \leq C\left(\int_{B_{r}} v^{q^{\prime}\left(p(x)-\theta^{-}\right)} d x\right)^{\frac{1}{q^{\prime}}}\left(\int_{B_{r}} v^{q \tau} d x\right)^{\frac{1}{q}} \\
& \leq C\left(1+\|v\|_{L^{q^{\prime} s}\left(B_{4 R}\right)}^{q^{\prime}\left(\theta^{+}-\theta^{-}\right)}\right)^{\frac{1}{q^{\prime}}}\left(\int_{B_{r}} v^{q \tau} d x\right)^{\frac{1}{q}}
\end{aligned}
$$

On the other hand and since $p(x)-1 \leq \delta(x)<p^{*}(x)$

$$
\begin{gathered}
\int_{B_{r}} \eta^{\theta^{+}-1} u^{\tau-\theta^{-}+1+\delta(x)} d x \leq \int_{B_{r}} v^{\tau-\theta^{-}+1+\delta(x)} d x \\
\leq C\left(\int_{B_{r}} v^{q^{\prime}\left(\delta(x)-\theta^{-}+1\right)} d x\right)^{\frac{1}{q^{\prime}}}\left(\int_{B_{r}} v^{q \tau} d x\right)^{\frac{1}{q}} \\
\left.\quad \leq C\left(1+\|v\|_{L^{q^{\prime} s}\left(B_{4 R}\right)}^{q^{\prime}\left(\theta^{+}\right)}\right)\right)^{\frac{1}{q^{\prime}}}\left(\int_{B_{r}} v^{q \tau} d x\right)^{\frac{1}{q}}
\end{gathered}
$$

Therefore, the second integral $I_{2}$ is estimated by (3).

In lemma 2.1 we take $u=v^{\frac{\tau}{\theta^{-}}} \eta^{\frac{\theta^{-}}{\theta^{-}}}$and we use the inequality:

$$
(a+b)^{p(.)} \leq 2^{p(.)-1}\left(|a|^{p(.)}+|b|^{p(.)}\right), \quad p(.) \geq 1,
$$

We obtain

$$
\begin{aligned}
& \left(\int_{B_{\rho}} v^{\frac{d \tau}{d-1}}\right)^{\frac{d-1}{d}} \leq C\left(\int_{B_{r}}\left(v^{\frac{\tau}{\theta^{-}}} \eta^{\frac{\theta^{-}}{\theta^{-}}}\right)^{\frac{d \theta^{-}}{d-1}} d x\right)^{\frac{d-1}{d}} \\
& \quad \leq C R^{\theta^{-}} \int_{B_{r}}\left|\nabla\left(v^{\frac{\tau}{\theta^{-}}} \eta^{\frac{\theta^{-}}{\theta^{-}}}\right)\right|^{\theta^{-}} d x \\
& \quad \leq C|\tau|^{\theta^{-}} \int_{B_{r}} \eta^{\theta^{+}} v^{\tau-\theta^{-}}|\nabla u|^{\theta^{-}} d x+C \int_{B_{r}} v^{\tau} \eta^{\theta^{+}-\theta^{-}}|\nabla \eta|^{\theta^{-}} d x
\end{aligned}
$$

Using inequality (4) we have 


$$
\begin{aligned}
& \left(\int_{B_{\rho}} v^{\frac{d \tau}{d-1}}\right)^{\frac{d-1}{d}} \leq C\left(\int_{B_{r}}\left(v^{\frac{\tau}{\theta^{-}}} \eta^{\frac{\theta^{-}}{\theta^{-}}}\right)^{\frac{d \theta^{-}}{d-1}} d x\right)^{\frac{d-1}{d}} \\
& \quad \leq C R^{\theta^{-}} \int_{B_{r}}\left|\nabla\left(v^{\frac{\tau}{\theta^{-}}} \eta^{\frac{\theta^{-}}{\theta^{-}}}\right)\right|^{\theta^{-}} d x \\
& \quad \leq C|\tau|^{\theta^{-}} \int_{B_{r}}\left(\eta^{\theta^{+}} u^{\tau-\theta^{-}}+u^{\tau-\theta^{-}+p(x)}|\nabla \eta|^{p(x)}+v^{\tau} \eta^{\theta^{+}-\theta^{-}}|\nabla \eta|^{\theta^{-}}\right) d x \\
& \quad+C \int_{B_{r}}\left(u^{\tau-\theta^{-}+p(x)}+\eta^{\theta^{+}-1} u^{\tau-\theta^{-}+1+\delta(x)}\right) d x \\
& \quad \leq C(1+|\tau|)^{\theta^{+}}\left(1+\|v\|_{L^{q^{\prime}}\left(B_{4 R}\right)}^{q^{\prime}\left(\theta^{+}-\theta^{-}\right)}\right)^{\frac{1}{q^{\prime}}}\left(\frac{r}{r-\rho}\right)^{\theta^{+}}\left(\int_{B_{r}} v^{q \tau} d x\right)^{\frac{1}{q}}
\end{aligned}
$$

Finally, since $\tau<0$ we have

$$
\Phi\left(v, q \tau, B_{r}\right) \leq C^{\frac{1}{|\tau|}}(1+|\tau|)^{\frac{p_{B_{4 R}}^{+}}{|\tau|}}\left(\frac{r}{r-\rho}\right)^{\frac{p_{B_{4 R}}^{+}}{|\tau|}} \Phi\left(v, \tau \frac{d}{d-1}, B_{\rho}\right)
$$

So the proof of lemma is achieved.

The proofs of the following results can be found in [11]. and [7], respectively

Lemma 3.6 Assume that $u$ is a nonnegative $p($.$) -supersolution of (1) in$ $B_{4 R}$ and $s>p_{B_{4 R}}^{+}-p_{B_{4 R}}^{-}$. Then there exist constants $q_{0}>0$ and $C$ depending on $d, p$, and $L^{q^{\prime} s}\left(B_{4 R}\right)$-norm of $u$ such that:

$$
\Phi\left(v, q_{0}, B_{3 R}\right) \leq C \Phi\left(v,-q_{0}, B_{3 R}\right)
$$

Theorem 3.7 (Weak Harnack inequality) Let u be a non-negative p(.)super-

solution of (1) inB $_{4 R}$ and $1<q<\frac{d}{d-1}$. Then

$$
\left(\int_{B_{2 R}} u^{q_{0}} d x\right)^{\frac{1}{q_{0}}} \leq C\left(\text { ess } \inf _{x \in B_{R}} u(x)+R\right)
$$

Where $q_{0}$ is the exponent from Lemma 3.5 and $C$ depends on $d, p, q$ and the $L^{q^{\prime} s}\left(B_{4 R}\right)$-norm of $u$ with $s>p_{B_{4 R}}^{+}-p_{B_{4 R}}^{-}$and all structure constants and functions of (H1),(H2) and (H3) hypothesis .

Theorem 3.8 Let $u$ be a non-negative $p($.$) -subsolution of (1) in B_{4 R}$ and $1<q<\frac{d}{d-1}$. Then

$$
\text { ess } \sup _{x \in B_{R}} u(x) \leq C\left(\int_{B_{2 R}} u^{t} d x\right)^{\frac{1}{t}}+R
$$

Where $t>0$ and $C$ depends on $d, p, q$ and the $L^{q^{\prime} s}\left(B_{4 R}\right)$-norm of $u$ with $s>$ $p_{B_{4 R}}^{+}-p_{B_{4 R}}^{-}$. 
To combine (9) and (10) we obtain the crucial theorem.

Theorem 3.9 (Harnack's inequality) Let u be a non-negative $p($.$) -solution$

of (1) in $\Omega$ and let $B_{4 R} \subset \Omega$ and $1<q<\frac{d}{d-1}$, and $s>p_{B_{4 R}}^{+}-p_{B_{4 R}}^{-}$with $0 \leq R \leq 1$. Then

$$
\text { ess } \sup _{x \in B_{R}} u(x) \leq C\left(\text { ess } \inf _{x \in B_{R}} u(x)+R\right)
$$

Where $C$ depends on $d, p, q$ and the $L^{q^{\prime} s}\left(B_{4 R}\right)$-norm of $u$ with and all structure constants and functions of (H1),(H2) and (H3) hypothesis .

\section{Conclusion}

We showed that $\mathrm{p}($.$) -solutions are locally bounded, locally bounded \mathrm{p}($.$) -supersolutions$ satisfy the weak Harnack inequality and locally bounded $\mathrm{p}($.$) -solutions satisfy$ Harnack's inequality. In the proof of Harnack's inequality the Caccioppoli estimate is used for the function $u+R$, where $\mathrm{R}$ is a radius of a ball.

\section{References}

[1] E. Acerbi, G. Mingione, Regularity results for a class of functionals with nonstandard growth, Arch. Ration. Mech. Anal. 156 (2) (2001) 121-140.

[2] Y. A. Alkhutov, The Harnack inequality and the Hölder property of solutions of nonlinear elliptic equations with a nonstandard growth condition, Differ. Uravn. 33 (12) (1997) 1651-1660, 1726.

[3] Y. A. Alkhutov and O. V. Krasheninnikova, Continuity at boundary points of solutions of quasilinear elliptic equations with a nonstandard growth condition, English translation in Izvestiya: Mathematics 68 (2004), no. $6,1063-1117$.

[4] A.Baalal \& A.Qabil, The p(.)-obstacle problem for quasilinear elliptic equations in sobolev spaces with variable exponent, International Journal of Applied Mathematics and Statistics, Vol. 48; Issue 18, 2013.

[5] L.Diening, P.Harjulehto, P.Hasto, M.Ruzicka, Lebesgue and Sobolev Spaces with Variable Exponents, Academic Press, New York, 2011.

[6] P. Hasto, Counter examples of regularity in variable exponent Sobolev spaces, Contemp. Math,367 (2005)133-143. 
[7] P.Harjulehto, Juha KinnunenT, Lukkari, Unbounded Supersolutions of Nonlinear Equations with Nonstandard Growth, Boundary Value Problems, Volume 2007, Article ID 48348, 20 pages doi:10.1155/2007/48348.

[8] X. L. Fan and D. Zhao, On the generalised Orlicz-Sobolev Space $\mathcal{W}^{k, p(x)}(\Omega)$, J. Gansu Educ. College12(1)(1998) 1-6.

[9] O. Ková(c)ik and J. Rákosnik, On spaces $L^{p(x)}$ and $W^{1, p(x)}$, Czechoslovak Math, J. 41(116)(1991), 592-618.

[10] J. Moser, On Harnack's theorem for elliptic differential equations, Comm. Pure Appl. Math.14 (1961), 577591.

[11] Olli. Toivanen, Harnack's inequality for general solutions with nonstandard growth, Ann. Acad. Sci. Fenn. Math., 37 (2012), 571-577.

[12] Yongqiang Fu, Yingying Shan, Removability of singularity for nonlinear elliptic equations with $\mathrm{p}(\mathrm{x})$-growth, Electronic Journal of Qualitative Theory of Differential Equations 2013, No. 58, 1-17.

[13] X. Zhang and X. Liu, The local boundedness and Harnack inequality of p(x)-Laplace equation. J. Math. Anal. Appl., 332:209-218, 2007.

[14] D. Zhao, W. J. Qiang and X. L. Fan, On generalized Orlicz spaces $L^{p(x)}(\Omega)$, J. Gansu Sci. 9(2) 1997 1-7.

[15] V. V. Zhikov, On Lavrentiev's phenomenon, Russian J. Math. Phys, 3(2) (1995) 249-269.

\section{Received: December 1, 2014}

